\title{
natureINSIGHT EXOPLANETS
}

18 September 2014 / Vol 513 / Issue No 7518

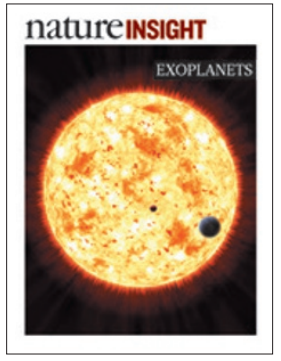

Cover illustration Nik Spencer

\section{Editor, Nature}

Philip Campbell

Publishing

Richard Hughes

Production Editor

Jenny Rooke

Art Editor

Nik Spencer

Sponsorship

Reya Silao

Production

lan Pope

Marketing

Steven Hurst

Editorial Assistant

Melissa Rose

The Macmillan Building 4 Crinan Street London N1 9XW, UK Tel: +44 (0) 2078334000 e: nature@nature.com

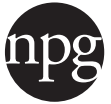

nature publishing group
I is hard to imagine now, and the younger people in the field will not remember this, but there was a period when the search for exoplanets had rather a bad reputation, based on a number of high-profile claims that were subsequently disproved. Although there was broad agreement, even by the 1980s, that planet formation ought to be a natural part of the star-formation process, at least for low-mass stars, we were still basing our assumptions on what we might find using the Solar System as a template.

On a late-summer morning in 1995, I picked up a new manuscript by Michel Mayor and Didier Queloz. After reading the paper, I thought, 'this looks pretty promising'. My next thoughts were, how could a planet be so close to its parent star - it seemed very unlikely that it could form there - and was such a planet stable against evaporation by stellar radiation? I picked up the phone to a colleague knowledgeable in such things, and the second question was rapidly answered in the affirmative. The first question is still a topic of research, although the two main options of disk migration and gravitational scattering emerged quite rapidly.

Nineteen years later, this collection of exoplanet papers reviews the state of the field. It is only fitting that Mayor, along with his co-authors Christophe Lovis and Nuno Santos, provide an overview of where we stand today.

Most known exoplanets were discovered, through planetary transits of the parent star's disk, by the Kepler space telescope. Jack Lissauer, Rebekah Dawson and Scott Tremaine assess the highlights of the mission.

Adam Burrows goes on to look at our current theoretical understanding of exoplanets and their atmospheres.

The path for Kepler was blazed by the under-rated Convection, Rotation and Planetary Transits (CoRoT) mission, with some help from Microvariability and Oscillation of Stars (MOST). Artie Hatzes fills us in on what those missions found.

Finally, the precise instruments needed to measure the radial-velocity shifts of stars as they and their planets co-orbit the system's centre of mass, along with present and future instruments to better characterize the planetary atmospheres, are reviewed and anticipated by Francesco Pepe, David Ehrenreich and Michael Meyer.

I hope that you enjoy this collection as much as I have enjoyed seeing history pass through my hands at Nature.

\section{Leslie Sage}

Astronomy Editor

\section{CONTENTS}

\section{REVIEWS}

328 Doppler spectroscopy as a path to the detection of Earth-like planets Michel Mayor, Christophe Lovis \& Nuno C. Santos

336 Advances in exoplanet science from Kepler

Jack J. Lissauer, Rebekah I. Dawson \& Scott Tremaine

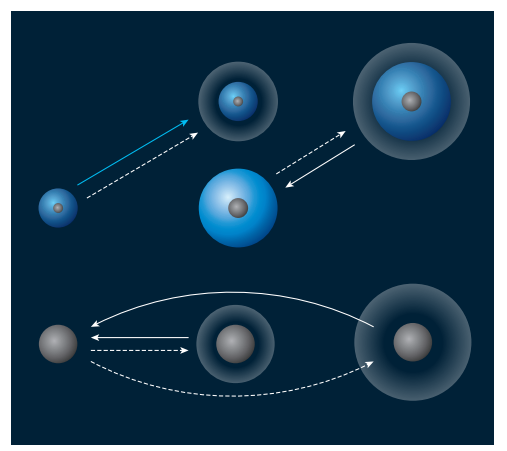

345 Highlights in the study of exoplanet atmospheres

Adam S. Burrows

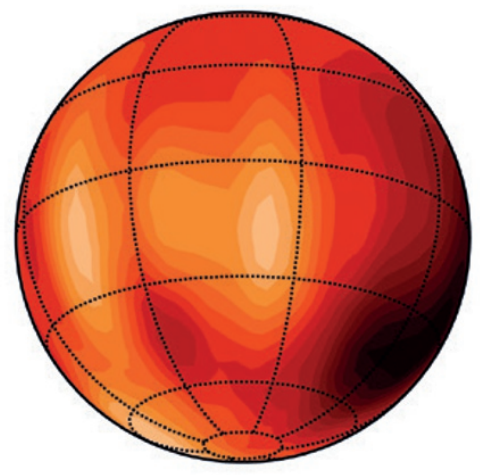

353 The role of space telescopes in the characterization of transiting exoplanets Artie P. Hatzes

358 Instrumentation for the detection and characterization of exoplanets Francesco Pepe, David Ehrenreich \& Michael R. Meyer 